PROCEEDINGS OF THE

AMERICAN MATHEMATICAL SOCIETY

Volume 130, Number 10, Pages 2999-3003

S 0002-9939(02)06449-3

Article electronically published on March 14, 2002

\title{
ERGODICITY OF THE ACTION OF THE POSITIVE RATIONALS ON THE GROUP OF FINITE ADELES AND THE BOST-CONNES PHASE TRANSITION THEOREM
}

\author{
SERGEY NESHVEYEV
}

(Communicated by David R. Larson)

\begin{abstract}
We study relatively invariant measures with the multiplicators $\mathbb{Q}_{+}^{*} \ni q \mapsto q^{-\beta}$ on the space $\mathcal{A}_{f}$ of finite adeles. We prove that for $\beta \in(0,1]$ such measures are ergodic, and then deduce from this the uniqueness of $\mathrm{KMS}_{\beta^{-}}$ states for the Bost-Connes system. Combining this with a result of Blackadar and Boca-Zaharescu, we also obtain ergodicity of the action of $\mathbb{Q}^{*}$ on the full adeles.
\end{abstract}

Bost and Connes $[\mathrm{BC}]$ constructed a remarkable $\mathrm{C}^{*}$-dynamical system which has a phase transition with spontaneous symmetry breaking involving an action of the Galois group $\operatorname{Gal}\left(\mathbb{Q}^{a b} / \mathbb{Q}\right)$, and whose partition function is the Riemann $\zeta$ function. In their original definition the underlying algebra arises as the Hecke algebra associated with an inclusion of certain $a x+b$ groups. Recently Laca and Raeburn [LR] L2] have realized the Bost-Connes algebra as a full corner of the crossed product algebra $C_{0}\left(\mathcal{A}_{f}\right) \rtimes \mathbb{Q}_{+}^{*}$. This new look at the system has allowed to simplify significantly the proof of the existence of KMS-states for all temperatures, and the classification of $\mathrm{KMS}_{\beta}$-states for $\beta>1$ [L1]. On the other hand, for $\beta \leq 1$ the uniqueness of $\mathrm{KMS}_{\beta}$-states implies ergodicity of the action of $\mathbb{Q}_{+}^{*}$ on $\mathcal{A}_{f}$ for certain measures (in particular, for the Haar measure). The aim of this note is to give a direct proof of the ergodicity, and then to show that the uniqueness of $\mathrm{KMS}_{\beta}$-states easily follows from it.

So let $\mathcal{P}$ be the set of prime numbers, $\mathcal{A}_{f}$ the restricted product of the fields $\mathbb{Q}_{p}$ with respect to $\mathbb{Z}_{p}, p \in \mathcal{P}, \mathcal{R}=\prod_{p} \mathbb{Z}_{p}$ its maximal compact subring, $W=$ $\mathcal{R}^{*}=\prod_{p} \mathbb{Z}_{p}^{*}$. The group $\mathbb{Q}_{+}^{*}$ of positive rationals is embedded diagonally into $\mathcal{A}_{f}$, and so acts by multiplication on the additive group of finite adeles. Then the Bost-Connes algebra $\mathcal{C}_{\mathbb{Q}}$ is the full corner of $C_{0}\left(\mathcal{A}_{f}\right) \rtimes \mathbb{Q}_{+}^{*}$ determined by the characteristic function of $\mathcal{R}$ [L2]. The dynamics $\sigma_{t}$ is defined as follows [L1]: it is trivial on $C_{0}\left(\mathcal{A}_{f}\right)$, and $\sigma_{t}\left(u_{q}\right)=q^{i t} u_{q}$, where $u_{q}$ is the multiplier of $C_{0}\left(\mathcal{A}_{f}\right) \rtimes \mathbb{Q}_{+}^{*}$ corresponding to $q \in \mathbb{Q}_{+}^{*}$. Then $([\mathrm{L} 1])$ there is a one-to-one correspondence between

Received by the editors November 28, 2000 and, in revised form, May 11, 2001.

1991 Mathematics Subject Classification. Primary 46L55; Secondary 28 D15.

This research was partially supported by Award No UM1-2092 of the Civilian Research \& Development Foundation. 
$\left(\beta, \sigma_{t}\right)$-KMS-states on $\mathcal{C}_{\mathbb{Q}}$ and measures $\mu$ on $\mathcal{A}_{f}$ such that

$$
\left.\mu(\mathcal{R})=1 \text { and } q_{*} \mu=q^{\beta} \mu \text { for all } q \in \mathbb{Q}_{+}^{*} \text { (i.e., } \mu\left(q^{-1} X\right)=q^{\beta} \mu(X)\right) .
$$

Namely, the KMS-state corresponding to $\mu$ is the restriction of the dual weight on $C_{0}\left(\mathcal{A}_{f}\right) \rtimes \mathbb{Q}_{+}^{*}$ to $\mathcal{C}_{\mathbb{Q}}$.

Note ([L1] $)$ that if $\beta>1$ and $\mu$ is a measure with property $(1 \beta)$, then

$$
\mu(W)=\prod_{p \in \mathcal{P}}\left(1-p^{-\beta}\right)=\frac{1}{\zeta(\beta)}>0,
$$

since $W=\mathcal{R} \backslash \bigcup_{p} p \mathcal{R}$. Moreover, the sets $q W, q \in \mathbb{Q}_{+}^{*}$, are disjoint, and their union is a set of full measure (since $\sum_{n \in \mathbb{N}} \mu(n W)=\frac{1}{\zeta(\beta)} \sum_{n \in \mathbb{N}} n^{-\beta}=1$ ). Thus there exists a one-to-one correspondence between probability measures on $W$ and measures on $\mathcal{A}_{f}$ satisfying $(1 \beta)[\mathrm{L} 1$. On the other hand, if $\beta \leq 1$, then $\mu(W)=0$.

Proposition. For $\beta \in(0,1]$ and any measure $\mu$ satifying $(1 \beta)$, the action of $\mathbb{Q}_{+}^{*}$ on $\left(\mathcal{A}_{f}, \mu\right)$ is ergodic.

Proof. Consider the space $L^{2}(\mathcal{R}, d \mu)$ and the subspace $H$ of it consisting of the functions that are constant on $\mathbb{N}$-orbits. In other words,

$$
H=\left\{f \in L^{2}(\mathcal{R}, d \mu) \mid V_{n} f=f, n \in \mathbb{N}\right\},
$$

where $\left(V_{n} f\right)(x)=f(n x)$. Since any $\mathbb{Q}_{+}^{*}$-invariant subset of $\mathcal{A}_{f}$ is completely determined by its intersection with $\mathcal{R}$, it suffices to prove that $H$ consists of constant functions. For this we will compute the action of the projection $P: L^{2}(\mathcal{R}, d \mu) \rightarrow H$ on functions spanning a dense subspace of $L^{2}(\mathcal{R}, d \mu)$.

Let $B$ be a finite subset of $\mathcal{P}$. Consider the projection $\pi_{B}: \mathcal{R} \rightarrow \prod_{p \in B} \mathbb{Z}_{p}$, and set $\mu_{B}=\left(\pi_{B}\right)_{*} \mu$. Then $L^{2}\left(\prod_{p \in B} \mathbb{Z}_{p}, d \mu_{B}\right)$ can be considered as a subspace of $L^{2}(\mathcal{R}, d \mu)$, and the union of these subspaces over all finite $B$ is dense in $L^{2}(\mathcal{R}, d \mu)$. The characters of $\prod_{p \in B} \mathbb{Z}_{p}^{*}$ span a dense subspace of $L^{2}\left(\prod_{p \in B} \mathbb{Z}_{p}^{*}, d \mu_{B}\right)$. Let $\mathbb{N}_{B}$ be the unital multiplicative subsemigroup of $\mathbb{N}$ generated by $p \in B$. Note that the sets $n \prod_{p \in B} \mathbb{Z}_{p}^{*}, n \in \mathbb{N}_{B}$, are disjoint, their union is a subset of $\prod_{p \in B} \mathbb{Z}_{p}$ of full measure (condition $(1 \beta)$ implies that the set $\left\{x \in \mathcal{R} \mid x_{p}=0\right\}$ has zero measure), and the operator $n^{-\beta / 2} V_{n}^{*}$ maps isometrically $L^{2}\left(\prod_{p \in B} \mathbb{Z}_{p}^{*}, d \mu_{B}\right)$ onto $L^{2}\left(n \prod_{p \in B} \mathbb{Z}_{p}^{*}, d \mu_{B}\right)$ for any $n \in \mathbb{N}_{B}$. Hence the functions $V_{n}^{*} \chi, n \in \mathbb{N}_{B}, \chi \in\left(\prod_{p \in B} \mathbb{Z}_{p}^{*}\right)^{\wedge}$, span a dense subspace of $L^{2}\left(\prod_{p \in B} \mathbb{Z}_{p}, d \mu_{B}\right)$. So we have to compute $P V_{n}^{*} \chi$. But if $g \in H$, then $\left(V_{n}^{*} \chi, g\right)=(\chi, g)$, whence $P V_{n}^{*} \chi=P \chi$. Thus we have only to compute $P \chi$.

For a finite subset $A$ of $\mathcal{P}$, let $H_{A}$ be the subspace consisting of the functions that are constant on $\mathbb{N}_{A}$-orbits, $P_{A}$ the projection onto $H_{A}$. Then $P_{A} \searrow P$ as $A \nearrow \mathcal{P}$. Set

$$
W_{A}=\prod_{p \in A} \mathbb{Z}_{p}^{*} \times \prod_{q \in \mathcal{P} \backslash A} \mathbb{Z}_{q} \subset \mathcal{R} .
$$

Note, as above, that $\bigcup_{n \in \mathbb{N}_{A}} n W_{A}$ is a subset of $\mathcal{R}$ of full measure. We assert that

$$
\left.P_{A} f\right|_{\mathbb{N}_{A} x} \equiv \frac{1}{\zeta_{A}(\beta)} \sum_{n \in \mathbb{N}_{A}} n^{-\beta} f(n x) \text { for } x \in W_{A}
$$


where $\zeta_{A}(\beta)=\sum_{n \in \mathbb{N}_{A}} n^{-\beta}=\prod_{p \in A}\left(1-p^{-\beta}\right)^{-1}$. Indeed, denoting the right hand part of (2) by $f_{A}$, for $g \in H_{A}$ we obtain

$$
\begin{aligned}
\left(f_{A}, g\right) & =\sum_{n \in \mathbb{N}_{A}} \int_{n W_{A}} f_{A}(x) \overline{g(x)} d \mu(x)=\sum_{n \in \mathbb{N}_{A}} n^{-\beta} \int_{W_{A}} f_{A}(x) \overline{g(x)} d \mu(x) \\
& =\zeta_{A}(\beta) \int_{W_{A}} f_{A}(x) \overline{g(x)} d \mu(x)=\sum_{n \in \mathbb{N}_{A}} n^{-\beta} \int_{W_{A}} f(n x) \overline{g(x)} d \mu(x) \\
& =\sum_{n \in \mathbb{N}_{A}} \int_{n W_{A}} f(x) \overline{g(x)} d \mu(x)=(f, g) .
\end{aligned}
$$

Returning to the computation of $P \chi$, we see that

$$
\left.P_{A} \chi\right|_{\mathbb{N}_{A} x} \equiv \frac{\chi(x)}{\zeta_{A}(\beta)} \sum_{n \in \mathbb{N}_{A}} n^{-\beta} \chi(n)=\chi(x) \prod_{p \in A} \frac{1-p^{-\beta}}{1-\chi(p) p^{-\beta}} \text { for } x \in W_{A} .
$$

Hence

$$
\|P \chi\|_{2}=\lim _{A \nearrow \mathcal{P}} \prod_{p \in A}\left|\frac{1-p^{-\beta}}{1-\chi(p) p^{-\beta}}\right| .
$$

If $\chi$ is trivial, then using (3) we see that $P_{A} \chi \equiv \prod_{p \in B}\left(1-p^{-\beta}\right)$ for all $A \supset B$, hence $P \chi$ is a constant. Suppose $\chi$ is non-trivial. The limit in (4) is an increasing function in $\beta$ on $(0,+\infty)$ (because each factor is increasing), which is equal to $|L(\beta, \chi)| \zeta(\beta)^{-1}$ for $\beta>1$, where $L(\beta, \chi)=\sum_{n=1}^{\infty} \chi(n) n^{-\beta}$ is the Dirichlet $L$ function corresponding to the number character $\chi[\underline{S}$. By elementary properties of Dirichlet series, $|L(\beta, \chi)|$ tends to a finite value as $\beta \rightarrow 1+0$, while $\zeta(\beta) \rightarrow \infty$. Thus $P \chi=0$.

Since the set of measures satisfying $(1 \beta)$ is convex and consists of ergodic measures, there exists at most one measure satisfying $(1 \beta)$. Such a measure does exist. In fact, for each $\beta \in(0,+\infty)$ there is a unique $W$-invariant measure $\mu_{\beta}$ satisfying

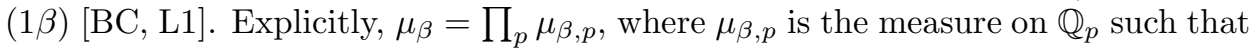
$\mu_{1, p}$ is the Haar measure $\left(\mu_{1, p}\left(\mathbb{Z}_{p}\right)=1\right)$, and

$$
\frac{d \mu_{\beta, p}}{d \mu_{1, p}}(a)=\frac{1-p^{-\beta}}{1-p^{-1}}|a|_{p}^{\beta-1} \text { for } a \in \mathbb{Q}_{p} .
$$

Let $\phi_{\beta}$ be the $\left(\beta, \sigma_{t}\right)$-KMS state on $\mathcal{C}_{\mathbb{Q}}$ corresponding to $\mu_{\beta}$. Then $\pi_{\phi_{\beta}}\left(\mathcal{C}_{\mathbb{Q}}\right)^{\prime \prime}$ is a factor, which is a reduction of the factor $L^{\infty}\left(\mathcal{A}_{f}, \mu_{\beta}\right) \rtimes \mathbb{Q}_{+}^{*}$. It is easy to describe its flow of weights $[\mathrm{CT}]$. Consider a standard measure space $X_{\beta}$ with the measure algebra consisting of $\mathbb{Q}_{+}^{*}$-invariant $\left(\lambda \times \mu_{\beta}\right)$-measurable subsets of $\mathbb{R}_{+} \times \mathcal{A}_{f}$, where $\lambda$ is the Lebesgue measure, and the flow $F_{t}^{\beta}$ on it coming from the action $t(x, a)=$ $\left(e^{-t / \beta} x, a\right)$ of $\mathbb{R}$ on $\mathbb{R}_{+} \times \mathcal{A}_{f}$. Then $F_{t}^{\beta}$ is ergodic by Proposition, and it is the flow of weights of the factors $L^{\infty}\left(\mathcal{A}_{f}, \mu_{\beta}\right) \rtimes \mathbb{Q}_{+}^{*}$ and $\pi_{\phi_{\beta}}\left(\mathcal{C}_{\mathbb{Q}}\right)^{\prime \prime}$.

Theorem ([BC] $)$. For $\beta \in(0,1], \mu_{\beta}$ is a unique measure satisfying $(1 \beta)$. The action of $\mathbb{Q}_{+}^{*}$ on $\left(\mathcal{A}_{f}, \mu_{\beta}\right)$ is ergodic, moreover, the action of $\mathbb{Q}^{*}$ on $\left(\mathcal{A}, \nu_{\beta}\right)$, where $\mathcal{A}=\mathbb{R} \times \mathcal{A}_{f}$ is the space of full adeles and $\nu_{\beta}=\lambda \times \mu_{\beta}$, is ergodic. Equivalently, $\phi_{\beta}$ is a unique $\left(\beta, \sigma_{t}\right)-K M S$ state on $\mathcal{C}_{\mathbb{Q}}$, and $\pi_{\phi_{\beta}}\left(\mathcal{C}_{\mathbb{Q}}\right)^{\prime \prime}$ is the hyperfinite factor of type $I I_{1}$. 
Proof. In view of the above description of the flow of weights, the factor $\pi_{\phi_{\beta}}\left(\mathcal{C}_{\mathbb{Q}}\right)^{\prime \prime}$ is of type $\mathrm{III}_{1}$ if and only if the action of $\mathbb{Q}_{+}^{*}$ on $\left(\mathbb{R}_{+} \times \mathcal{A}_{f}, \lambda \times \mu_{\beta}\right)$ is ergodic, or equivalently, the action of $\mathbb{Q}^{*}$ on $\left(\mathcal{A}, \nu_{\beta}\right)$ is ergodic.

To prove the ergodicity, first note that the action of $W$ on $X_{\beta}$ is ergodic. Indeed, the induced flow on $X_{\beta} / W$ is the flow of weights of the factor

$$
L^{\infty}\left(\mathcal{A}_{f} / W, \mu_{\beta}\right) \rtimes \mathbb{Q}_{+}^{*}=\left(L^{\infty}\left(\mathcal{A}_{f}, \mu_{\beta}\right) \rtimes \mathbb{Q}_{+}^{*}\right)^{W} .
$$

It is easy to see $(\overline{\mathrm{BC}})$ that this factor is ITPFI with eigenvalue list

$$
\left\{p^{-n \beta}\left(1-p^{-\beta}\right) \mid n \geq 0\right\}_{p \in \mathcal{P}} .
$$

Hence it is of type $\mathrm{III}_{1}$ by $[\mathrm{B}]$ (see also [BZ]). Thus its flow of weights is trivial, i.e. the action of $W$ on $X_{\beta}$ is ergodic. Since $W$ is compact, the action is transitive. So we may identify $X_{\beta}$ with $W / W_{\beta}$ for some closed subgroup $W_{\beta}$ of $W$. Then the flow $F_{t}^{\beta}$ is given by a continuous one-parametric subgroup of $W / W_{\beta}$. Since $W / W_{\beta}$ is totally disconnected, this one-parametric subgroup is trivial, and since the flow is ergodic, $W_{\beta}=W$. Thus $X_{\beta}$ is singlepoint, and the action of $\mathbb{Q}_{+}^{*}$ on $\left(\mathbb{R}_{+} \times \mathcal{A}_{f}, \lambda \times \mu_{\beta}\right)$ is ergodic.

Remarks. (i) In order to prove that $\phi_{\beta}$ is a unique $\mathrm{KMS}_{\beta}$-state, it is enough to know that $\mu_{\beta}$ is ergodic. Indeed, this means that $\phi_{\beta}$ is an extremal $\mathrm{KMS}_{\beta}$-state. Since $\phi_{\beta}$ is a unique $W$-invariant $\mathrm{KMS}_{\beta}$-state, we can argue as in the proof of [BC] Theorem 25]: if $\psi$ is an extremal $\mathrm{KMS}_{\beta}$-state, then

$$
\int_{W} w_{*} \psi d w=\phi_{\beta}
$$

Since $\mathrm{KMS}_{\beta}$-states form a simplex, we conclude that $\psi=\phi_{\beta}$.

Thus, the uniqueness and the assertion about the type for $\mathrm{KMS}_{\beta}$-states follow easily from ergodicity of the action of $\mathbb{Q}^{*}$ on $\left(\mathcal{A}, \nu_{\beta}\right)$.

(ii) A slight modification of the argument in the proof of the Theorem gives the following general result, apparently well-known to specialists: if $M$ is a factor and $G$ a compact totally disconnected group acting on $M$ such that $M^{G}$ is a factor of type $\mathrm{III}_{1}$, then $M$ is also of type $\mathrm{III}_{1}$.

\section{ACKNOWLEDGEMENT}

The author is grateful to Marcelo Laca, Sergey Sinelshchikov and Yoshimichi Ueda for helpful discussions.

\section{REFERENCES}

[B] Blackadar B., The regular representation of restricted direct product groups, J. Func. Anal. 25 (1977), 267-274. MR 55:12860

[BZ] Boca F.P., Zaharescu A., Factors of type III and the distribution of prime numbers, Proc. London Math. Soc. 80 (2000), 145-178. MR 2000j:11133

[BC] Bost J.-B., Connes A., Hecke algebras, type III factors and phase transitions with spontaneous symmetry breaking in number theory, Selecta Math. (New Series) 1 (1995), 411-457. MR 96m:46112

[CT] Connes A., Takesaki M., The flow of weights on factors of type III, Tôhoku Math. J. 29 (1977), 473-575. MR 82a:46069a

[L1] Laca M., Semigroups of *-endomorphisms, Dirichlet series, and phase transitions, J. Func. Anal. 152 (1998), 330-378. MR 99f:46097

[L2] Laca M., From endomorphisms to automorphisms and back: dilations and full corners, J. London Math. Soc. 61 (2000), 893-904. CMP 2000:14 
[LR] Laca M., Raeburn I., A semigroup crossed product arising in number theory, J. London Math. Soc. 59 (1999), 330-344. MR 2000g:46097]

[S] Serre J.-P. Cours d'arithmétique. P.U.F. Paris, 1970. MR 41:138

Institute for Low Temperature Physics \& Engineering, 47 Lenin ave, Kharkov 310164 , UKRAINE

Current address: Matematisk Institutt, P.B. 1053 Blindern, 0316 Oslo, Norway

E-mail address: neshveyev@hotmail.com 